\title{
STATUS OF THE TAIWAN LIGHT SOURCE
}

\author{
R.C. Sah", J.R. Chen, P.J. Chou, and K.K. Lin \\ Synchrotron Radiation Research Center, Hsinchu, Taiwan
}

\begin{abstract}
The Taiwan Light Source (TLS) is a third-generation synchrotron light source located at the Synchrotron Radiation Research Center (SRRC) in Taiwan. The TLS is now operating routinely for user experiments, and the highest priorities are increasing user beam time, increasing machine reliability, and improving the stability of the stored electron beam. Many current machine upgrades are intended to improve beam stability: the longitudinal feedback system, orbit feedback systems, radiofrequency-system improvements (better higherorder-mode performance), and utilities upgrades. In addition, a major improvement program to increase the stored beam current is being planned.
\end{abstract}

\section{INTRODUCTION}

The Taiwan Light Source has served users for several years [1]. Recently, a great deal of the laboratory effort and budget has been devoted to improving the capability of the research center to support a wide spectrum of user groups. That is, the construction of insertion devices, photon beamlines, and experimental end stations has taken first priority.

As the construction of user facilities is starting to slow down a little, greater resources are starting to become available for accelerator-system repairs and upgrades. Therefore, changes in machine operations and machine upgrade programs are focused on the four following goals:

- To increase user beam time,

- To increase beam reliability,

- To improve the stability of the photon beams, and

- To increase the performance capabilities of the TLS.

\section{USER BEAM TIME}

The amount of beam time available for users has increased in recent years, because the number of days of machine operation has been steadily increased. Earlier, the Taiwan Light Source was operated for five days out of every week. In 1997, the operations were increased to six days per week, and now the machine is operated seven days per week. A three-day maintenance period requires a machine shutdown once per month, and long shutdowns are scheduled as needed.

\footnotetext{
"Email: sah@ srrc.gov.tw
}

Mondays are normally reserved for machine studies, and the change-overs between experiments are scheduled for the same time. In addition, many owl shifts are used for machine studies.

\section{BEAM RELIABILITY}

The overall reliability of the TLS has improved markedly over the past few years. During 1996 and 1997, the machine reliability was approximately $89 \%$. Last year, however, the reliability increased to $96 \%$. It is true that one year does not represent a great deal of statistics in terms of machine reliability; however, we believe that this improvement is indeed real.

First of all, problem areas, such as RF transmitters, have been upgraded both in terms of the equipment and in terms of the maintenance. Secondly, reorganizations of the maintenance staff have improved the maintenance of some systems, such as the injector. Lastly, some systems, which have started to suffer from ageing and performance degradation (such as the conventional utilities systems - cooling water, etc.) have undergone significant repairs.

\section{BEAM STABILITY}

Stable photon beams are a crucial requirement of most user groups, and considerable effort is being devoted to improving the beam stability. Up to now, much of the effort has focused on first improving the stability of the stored electron beam.

\subsection{Sources of Noise and Instability}

Sources of noise and beam instability were studied in the TLS, and the most serious problems have been resolved. Worn-out bearings for air-conditioning-system fans were found to be a source of vibration, and they have been replaced.

Also, the ongoing upgrade program for the conventional utilities (cooling water systems, air conditioning system, etc.) has improved the performance capabilities of these systems. The temperature regulation of these systems has been very significantly improved, and the system capacities have been increased to meet growing requirements.

\subsection{Orbit Stability}

A global feedback system has been developed to control overall motions of the electron-beam orbit, and a local 
feedback system has been developed to control the orbit in local regions of the machine.

\subsection{Other Feedback Systems}

A transverse feedback system has been developed to suppress transverse instabilities, and a bunch-by-bunch longitudinal feedback system is being built to suppress longitudinal instabilities. In particular, the TLS is troubled sometimes by longitudinal coupled-bunch instabilities, which are very difficult to control. For the time being, the use of RF modulation, at about twice the synchrotron frequency, excites parametric resonances and helps to control these beam instabilities.

\section{UPGRADE PROJECT}

A new machine-upgrade program is being undertaken to increase the performance capabilities of the TLS.

\subsection{Project Goals}

The "500 mA Upgrade Project" is a major upgrade program with the following goals:

- To provide photon beams with significantly better stability, and

- To store up to $500 \mathrm{~mA}$ of electron beam, while maintaining the beam lifetime.

\subsection{Approach}

At this time, among the most troublesome causes of photon-beam instabilities in the TLS are the longitudinal coupled-bunch instabilities of the stored electron beam, as discussed above. These coupled-bunch instabilities are caused by the higher-order modes (HOMs) of the radiofrequency cavities, so we plan to replace the existing DORIS cavities with a new cavity, which has much weaker HOMs. Therefore, the major components of the $500 \mathrm{~mA}$ Upgrade Project are the following:

- A new RF cavity with reduced HOMs to provide improved beam stability and with the capability to store $2 \mathrm{X}$ higher beam current,

- Other RF system improvements to accommodate 2X higher stored beam current,

- Third-harmonic (Landau) cavities to lengthen bunch lengths by $2 \mathrm{X}$, and

- Other machine improvements, such as to the vacuum system and to the utilities systems, to accommodate operation at higher beam current.

At this time, a number of advanced RF cavity designs already exist in various accelerator laboratories around the world. Most of these designs have been developed for high-energy-physics accelerator applications.
Only one or two new RF cavities need to be installed in the storage ring of the TLS, so our needs are relatively modest. We believe that the cost and technical risk of new cavity development are not warranted for so few cavities, so our approach will be to acquire existing technology from other laboratories

\subsection{RF System Requirements}

The upgraded RF system should meet the following requirements:

- Deliver $100 \mathrm{~kW}$ of RF power to the beam at 500 $\mathrm{mA}$, which is enough power to accommodate the eventual addition of superconducting bend magnets or of superconducting wigglers,

- Provide additional RF power for cavity dissipation and operational safety margin, and

- Provide a gap voltage of 1.2 MV.

\subsection{Cavity Selection}

Advanced RF cavity designs have been studied at SRRC for well over a year, and we have recently selected the Cornell superconducting RF cavity [2].

We are planning a two-phased approach. Stable operation will be provided in Phase 1 , using one new RF cavity powered by one of the existing $60 \mathrm{~kW}$ transmitters. Additional RF power will be installed to provide $500 \mathrm{~mA}$ operation in Phase 2.

A second RF cavity will be purchased and kept in readiness on a test stand. The ability to install a functional cavity on short notice will provide maximum machine reliability.

\subsection{Project Status}

The current goal is to sign a purchase contract for the new superconducting cavities by mid-1999, so that the first phase of this upgrade project can be completed by the year 2002. The staff at CESR (Cornell University) has generously agreed to help with the technology transfer.

\section{REFERENCES}

[1] Y.C. Liu, et. al., "Operational Experience at SRRC", PAC"97, Vancouver, Canada, May 12-16, 1997, p.847.

[2] S. Belomestnykh, et. al., "Development of Superconducting RF for CESR", PAC'97, Vancouver, Canada, May 12-16, 1997, p.3075. 
Proceedings of the 1999 Particle Accelerator Conference, New York, 1999 